\title{
Development of a rapid field-applicable molecular diagnostic for knockdown resistance ( $k d r)$ markers in An. gambiae
}

\author{
Vera T. Unwin', Shaun Ainsworth², Emily J. Rippon', El Hadji Amadou Niang ${ }^{3}$, Mark J. I. Paine',
} David Weetman ${ }^{1 *}$ and Emily R. Adams ${ }^{1}$

\begin{abstract}
Background: The spread of insecticide resistance (IR) is a major threat to vector control programmes for mosquitoborne diseases. Early detection of IR using diagnostic markers could help inform these programmes, especially in remote locations where gathering reliable bioassay data is challenging. Most current molecular tests for genetic IR markers are only suitable for use in well-equipped laboratory settings. There is an unmet need for field-applicable diagnostics.

Methods: A single-cartridge test was designed to detect key IR mutations in the major African vector of malaria, Anopheles gambiae. Developed on the portable, rapid, point-of-care compatible PCR platform - Genedrive ${ }^{\circledast}$ (genedrive ${ }^{\circledast}$ plc), the test comprises two assays which target single nucleotide polymorphisms (SNPs) in the voltage gated sodium channel (VGSC) gene that exert interactive effects on knockdown resistance (kdr): L1014F, L1014S and N1575Y.

Results: Distinct melt peaks were observed for each allele at each locus. Preliminary validation of these assays using a test panel of 70 An. gambiae samples showed complete agreement of our assays with the widely-used TaqMan assays, achieving a sensitivity and specificity of $100 \%$.

Conclusion: Here we show the development of an insecticide resistance detection assay for use on the Genedrive ${ }^{\circledR}$ platform that has the potential to be the first field-applicable diagnostic for $k d r$.
\end{abstract}

\section{Background}

Control programmes for vector-borne diseases, such as malaria, are heavily reliant on the use of insecticides to reduce vector populations. The use of insecticide-treated nets (ITNs), long-lasting insecticidal nets (LLINs) and indoor residual spraying (IRS) plays a major role in the overall reduction in global malaria burden. [1]

Until recently, all ITNs and LLINs were formulated with pyrethroid insecticides [2]. The new development of a chlorfenapyr-based LLN offers a potential alternative, since resistance to pyrethroids is now widespread and has been reported in numerous mosquito species [3].

Knockdown resistance mutations $(k d r)$ in the para voltage-gated sodium channel $(\mathrm{Vgsc})$ of neurones are

\footnotetext{
* Correspondence: david.weetman@lstmed.ac.uk

${ }^{1}$ Liverpool School of Tropical Medicine, Pembroke Place, L3 5QA, Liverpool, UK

Full list of author information is available at the end of the article
}

one of the principle mechanisms of resistance to pyrethroids and dichlorodiphenyltrichloroethane (DDT) in insects, resulting in an increased tolerance to insecticide exposure, compromising vector mortality [4]. Three mutations in the Vgsc gene are linked to pyrethroid and DDT resistance in the principal African vectors of malaria: Anopheles gambiae, An. coluzzii and An. arabiensis. Most common are two mutations to the leucine residue at position 1014 in the wild-type Vgsc-1014 [L1014F (TTA > TTT) and L1014S (TTA > TCA)], located in the hydrophobic segment S6 of domain II (IIS6) resulting in a conformational change preventing access of the insecticide to the active site VGSC protein [5]. The L1014F ('F') mutation is the most common $k d r$ mutation across diverse insect taxa [6]. L1014S ('S') was previously only found in mosquitoes and has recently been detected in the visceral leishmaniasis vector Phlebotomus argentipes [7]. Although first detected in West and East 
Africa, $\mathrm{F}$ and $\mathrm{S}$ mutations have both been detected across the continent and sometimes co-occur [8]. The mutations do not occur on the same haplotypes, but where both alleles are present, effects on $k d r$ are additive [7, 9]. In An. gambiae and An. coluzzii, a third mutation in the Vgsc- Asp1575Tyr (ATA > ATT) has been found in West Africa [10]. The 1575Y ('Y') mutation is only present on the $\mathrm{F}$ haplotype and acts as an amplifier of resistance to both pyrethroids and DDT $[10,11]$.

Existing diagnostics for $k d r$ markers, i.e. allele-specific PCR (ASPCR), HOLA, SSOP-ELISA, PCR-Dot Blot, FRET/MCA, TaqMan and HRM, were recently compared to a new SimpleProbe ${ }^{\circ}$ RT-PCR/melt curve assay in Culex quinquefasciatus. The melt curve assay was found to be cheaper, faster and more reliable than alternatives [12]. Melt curve assays are designed on the principle that a sequence-specific probe will dissociate away from a DNA duplex at a characterising melting temperature $(\mathrm{Tm})$. In the presence of a mismatched sequence, probe-binding is less efficient, reducing the $\mathrm{Tm}$, and causing a characteristic Tm shift.

Although melt curve-based techniques have advantages over other PCR-based methods, they still require expensive, mains-powered real-time-PCR platforms and skilled technicians [12]. Furthermore, they have only been validated using purified DNA. There is a need for simpler field-applicable molecular tests that could be used in the resource-limited settings where vector control programmes are commonly employed.

Genedrive $^{\circ}$ is a molecular diagnostics platform that utilises PCR with detection of a fluorescent reporter dye. The total run time of the system ranges from 45 to 90 min depending on assay, and can be utilised direct from the electricity mains or via a portable uninterruptable power supply (UPS) which also functions as a battery. While existing Genedrive ${ }^{\bullet}$ tests target pathogens (HCV [13], MTb [14]) and pharmacogenomic mutations (IL28B [15]) from such diverse clinical samples as plasma, sputum and buccal swabs, respectively, the technology has not been adopted for use with insects.

\section{Methods}

\section{Primer and probe design}

Two separate assays were designed to target three individual SNPs within the Vgsc gene, the first to discriminate between the two mutations at the L1014 locus and the other at the $1575 \mathrm{Y}$ locus.

Dual-labelled fluorescent, HyBeacon-type molecular probes were designed against the L1014F and $1575 \mathrm{Y} \mathrm{mu-}$ tation sequences. The probes were designed to dissociate from the amplicon at around $62{ }^{\circ} \mathrm{C}$, whereas mismatched pairing would yield a reduced Tm shift $>2{ }^{\circ} \mathrm{C}$. The Tm of either probe was predicted in silico using OligoAnalyzer 3. 1 software (https://www.idtdna.com/calc/analyzer) before empirical determination on the LightCycler480 (Roche Applied Science, Penzberg, Germany) (Table 1). Initial screening experiments were carried out on this higherthroughput platform before transfer to the Genedrive following optimisation. The NCBI-BLAST software was used to design primers and probes specific to the $A n$. gambiae complex. Probes were obtained from ATD Bio (Southampton, UK) and primers from Eurofins (Ebersberg, Germany).

\section{Mosquitoes}

The following mosquitoes and DNA samples were used for the initial optimisation of the assays:

(i) $K d r$-susceptible, (S-form) An. gambiae (s.s.) (wildtype) and $k d r$-resistant, (M-form) An. coluzzii (F/F) mosquitoes (Kisumu and VK7 laboratory reference strains, respectively) were provided by the Liverpool Insecticide Testing Establishment (LITE) at the Liverpool School of Tropical Medicine.

(ii) $K d r$-resistant (S/S) An. arabiensis mosquitoes and dual $k d r$ - resistant (FY/FY, FF/FY), pre-extracted $A n$. arabiensis mosquito DNA came from recent field collections in Senegal, location and sampling details of which will be provided elsewhere.

(iii) A mixed-population panel of An. gambiae (s.s.) DNA of known genotypes provided from samples archived at LSTM.

\section{TaqMan reference standard}

Three separate TaqMan assays are routinely used for genotyping F [16], S [16] and Y [10] mutants. Here, we used these tests as reference standards, performed exactly as previously published $[10,16]$. Genotypes of all mosquitoes were confirmed using these established TaqMan genotyping assays.

\section{Lysate preparation}

The Genedrive ${ }^{\bullet}$ assay was optimised for use with crude mosquito-leg lysates, although N/Y and $\mathrm{Y} / \mathrm{Y}$ templates were only available as archived extracted DNA samples.

Table 1 Primer and probe selections following RT-PCR melt curve screening

\begin{tabular}{ll}
\hline Primer/probe & Sequence $\left(5^{\prime}-3^{\prime}\right)$ \\
\hline 1014 Forward primer & TCCCCGACCATGATCTGCCAA \\
1014 Reverse primer & GCACCTGCAAAACAATGTCATGTAA \\
1014 Probe & MGGAAATTITGTCGFAAGTAAFGCAAP \\
1575 Forward primer & AAAGAAAGCTGGTGGATCGC \\
1575 Reverse primer & TGAAAACACTAACCCTTGGACGA \\
1575 Probe & MTATTATGCAAFGAAAAAAAFGGGTP
\end{tabular}

$\mathrm{F}, \mathrm{M}$ and $\mathrm{P}$ (in bold) denote a fluorescein labelled deoxythymidine base, a trimethoxystilbene and propanol conjugate, respectively. 
For all the other genotypes, single mosquito legs were incubated in $100 \mu \mathrm{l}$ nuclease-free water at $95^{\circ} \mathrm{C}$ for $20 \mathrm{~min}$.

\section{DNA extraction and quantification}

For comparisons of the limit of detection (LOD) of the assay using purified DNA versus crude lysate, total genomic DNA was extracted from $\mathrm{L} / \mathrm{L}$ and $\mathrm{F} / \mathrm{F}$ mosquitoes using the Qiagen blood and tissue kit according to the manufacturer's protocol (Qiagen, Hilden, Germany). Extracted DNA was quantified using the Qubit dsDNA high sensitivity kit according to the manufacturer's protocol (Thermo Fisher, UK).

\section{The Genedrive ${ }^{\circledR}$ platform}

Details of the Genedrive ${ }^{\bullet}$ platform are published in Duffy et al. [15]. In brief, the device uses a single wavelength optical system (400-470 nm LEDs, $535 \mathrm{nM}$ photodiodes) to read a PCR test cartridge comprised of 3 reaction wells. Additional technical specifications are available at https://www. Genedrive.com/Genedrive-system/documentation.php.

\section{Genedrive $^{\circledR}$ assay optimisation}

Each reaction contained $10 \mu \mathrm{l}$ of lysate or extracted DNA template, $0.2 \mu \mathrm{M}$ of the probe, $0.1 \mu \mathrm{M}$ of forward primer, $4 \mu \mathrm{M}$ of reverse primer (Table 1 ) in a total reaction volume of $20 \mu \mathrm{l}$ of the following: $1 \mathrm{mM} \mathrm{MgCl}_{2}, 0.1$ mM dNTPs, $12.5 \mathrm{mM}$ Tris (pH 8.5), $\mathrm{KCl} 62.5 \mathrm{mM}$, BSA $0.5 \mathrm{mg} / \mathrm{ml}$, GoTaqMDx $0.075 \mathrm{U} / \mu \mathrm{l}$ and $0.25 \mu \mathrm{l}$ Excipient (GE Healthcare, Amersham, UK). Several primer/probe sets were screened before the final sets were selected (shown in Table 1). Initial optimisations on the LightCycler480 were carried out under the following cycling conditions: $95{ }^{\circ} \mathrm{C}$ for $10 \mathrm{~min}$, followed by 50 cycles of amplification at $95{ }^{\circ} \mathrm{C}$ for $10 \mathrm{~s}$, annealing at $62{ }^{\circ} \mathrm{C}$ for 10 $\mathrm{s}$, an extension at $72{ }^{\circ} \mathrm{C}$ for $10 \mathrm{~s}$, and a final extension at $72{ }^{\circ} \mathrm{C}$ for $1 \mathrm{~min}$. This was followed by a melt step increasing the temperature from 42 to $95{ }^{\circ} \mathrm{C}$ in $0.5{ }^{\circ} \mathrm{C}$ increments with continuous fluorescence acquisition. Genedrive $^{\circledast}$ platform heats and cools rapidly allowing thermocycling for less than $1 \mathrm{~s}$ and resulting in a short run time of $50 \mathrm{~min}$. Final reaction conditions used were: 50 cycles of amplification at $95{ }^{\circ} \mathrm{C}$ for $0 \mathrm{~s}$ (allowed to reach $95{ }^{\circ} \mathrm{C}$ only) before reducing the temperature to $62{ }^{\circ} \mathrm{C}$ for $10 \mathrm{~s}$, and then raising it to $72{ }^{\circ} \mathrm{C}$ for $0 \mathrm{~s}$ (again reaching that temperature only momentarily), followed by a final melt step increasing the temperature from $42{ }^{\circ} \mathrm{C}$ to $80{ }^{\circ} \mathrm{C}$ in $0.5{ }^{\circ} \mathrm{C}$ increments with continuous fluorescence acquisition, followed by a cooling to $40{ }^{\circ} \mathrm{C}$ for $60 \mathrm{~s}$.

\section{Analytical accuracy}

A randomly-chosen panel of 70 An. gambiae DNA samples was compiled from previously-collected and
TaqMan-genotyped, mosquito samples, and 10 notemplate controls were added. The operator of the Genedrive ${ }^{ø}$ assay was blinded to the genotypes of the panel.

Sensitivity of the 1014 assay was assessed using: (i) crude lysate and (ii) normalised DNA, from individual mosquitoes. DNA was tested at $1 \mathrm{ng} / \mu \mathrm{l}, 100 \mathrm{pg} / \mu \mathrm{l}$ and $10 \mathrm{pg} / \mu \mathrm{l}$. Crude lysate on the other hand was tested following dilution factors of: 20, 50 and 100, as DNA concentration could not be estimated in crude lysate owing to interference in absorption by liberated proteins. These dilution series were selected based on the amount of DNA a typical extraction might yield from a single mosquito.

\section{Pooling mosquitoes}

Mosquito pools contained a single F/F mosquito with either 3, 5 or 7, L/L mosquitoes. Crude lysates for these pools were obtained as described above following the addition of $100 \mu \mathrm{l}$ nuclease-free water per mosquito.

\section{Pooling crude lysates}

Lysate pools were constructed by mixing $10 \mu \mathrm{l}$ of crude lysate from individually lysed mosquitoes in the following $\mathrm{F} / \mathrm{F}$ to $\mathrm{L} / \mathrm{L}$ ratios: $1: 1,1: 3$ and $1: 4$.

\section{Pooling DNA}

Extracted DNA samples were first diluted in nucleasefree water to a working concentration of $1 \mathrm{ng} / \mu \mathrm{l}$. 'DNA pools' were generated in ratios of $1 / 4,1 / 5,1 / 6,1 / 7$ of $\mathrm{F} /$ F:L/L DNA.

\section{Results}

Primer and probe selection

Candidate primers were screened using WT mosquito lysate as template and the best pair was selected based on peak height fluorescence. Positive control DNA for each genotype was used to screen candidate probes and selection of the final probe was made according to largest observed $\mathrm{Tm}$ shifts $\left({ }^{\circ} \mathrm{C}\right)$ between genotypes, in addition to highest peak fluorescence (not shown). Selected primers and probes are shown in Table 1.

\section{Detection of $k d r$ alleles}

Using the Genedrive ${ }^{ø}$ platform, all genotypes were determined based on the presence of Tm specific peaks. Discernible melt peaks were observed for all alleles in both the 1014 or 1575 assays as highlighted in Fig. 1. In each case the peaks between wild-type and mutant were separated by Tm shifts of at least $2{ }^{\circ} \mathrm{C}$. (Fig. 2a-d) In comparison to extracted DNA, average Tm peaks of $\mathrm{L}$ and $\mathrm{F}$ alleles were slightly higher in lysates (Table 2). 


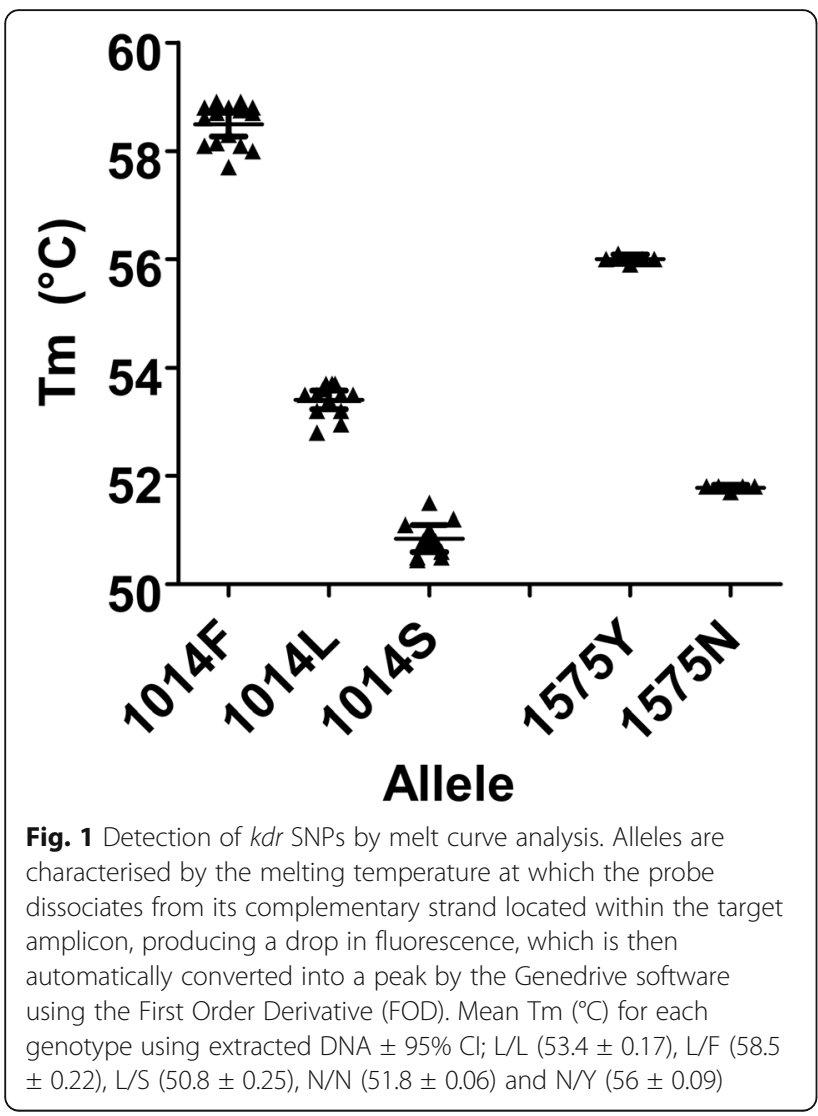

\section{Limits of detection}

The sensitivity of the 1014 assay when using individual mosquitoes was tested using: (i) extracted DNA at $1 \mathrm{ng} / \mu \mathrm{l}, 100 \mathrm{pg} / \mu \mathrm{l}$ and $10 \mathrm{pg} / \mu \mathrm{l}$ and (ii) crude lysate at dilutions $1 / 20,1 / 50$ and $1 / 100$. Figure 3 shows distinct Tm peaks (Tm shift between alleles $\geq 2{ }^{\circ} \mathrm{C}$ with FOD $\geq 100$ ) detected across all dilutions using DNA template; however, the F-peak starts to drop when using lysates. This suggests pooling of lysates is less sensitive than pooling DNA.

Sensitivity and specificity was determined by screening a panel of $A n$. gambiae (s.s.) DNA samples of known genotype identified using TaqMan assays as a reference standard. Table 3 shows that both 1014 and 1575 tests had $100 \%$ sensitivity and specificity.

\section{Detection of genotypes in mosquito pools}

To increase throughput, the sensitivity of the 1014 assay using pools of mosquitoes was investigated. These included: (i) pooling mosquitoes for lysate preparation and (ii) pooling lysates obtained from individual mosquitoes. Mosquito pools contained a single homozygous mutant F/F mosquito with either 3, 5 or 7 homozygous WT L/L mosquitoes. Lysates for these pools were generated as described earlier followed by the addition of $100 \mu \mathrm{l}$ nuclease-free water per mosquito.
Pooling lysates from individual mosquitoes resulted in poor detection of the $\mathrm{F}$ allele when diluted in a background of L alleles; a theoretical pool of 2 mosquitos (a single L/F heterozygote and an L/L homozygote) failed to produce a discernible peak (data not shown). Using extracted and normalised DNA significantly improved pooling results. Using $1 \mathrm{ng} / \mu \mathrm{l}$ of DNA, ratios of $1 / 4,1 / 5$, $1 / 6,1 / 7$ of homozygous F/F: L/L DNA were tested. Figure 4 shows example melt curves of these pools. Results show two discernible peaks at 58.8 and $53.6{ }^{\circ} \mathrm{C}$, corresponding to the $\mathrm{F}$ and $\mathrm{L}$ peaks, are observed at a $1 /$ 7 ratio (equivalent to a pool of 4 mosquitoes: one $\mathrm{F} / \mathrm{F}$ and three $\mathrm{L} / \mathrm{L}$ mosquitoes). Since the Genedrive ${ }^{\oplus}$ cartridge contains 3 wells, there is potential for 12 DNA samples to be screened in one run.

\section{Discussion}

Here we have developed a method using the Genedrive ${ }^{\circ}$ platform for the detection of three mutations that indicate insecticide resistance at the 1014 and 1575 loci in An. gambiae. Genedrive ${ }^{\circledast}$ uses end-point melt analysis to give rapid results with limited sample pre-processing, $[14,15]$ and is shown to be highly accurate when tested on a panel of 80 samples. Although wet reagents were used throughout this method development work, like all other commercially-available Genedrive ${ }^{\curvearrowleft}$ tests (HCV [13], MTb [14] and IL-28B [15]), the final optimised formulation will be lyophilised within the cartridges so that only the addition of template is required, and also eliminating the need for any cold chain storage. Additionally, the Genedrive ${ }^{\varpi}$ platform is very easy to use with only a single button required for its operation thereby simplifying the workflow and reducing the need for skilled operators. The Genedrive ${ }^{\bullet}$ platform is portable weighing less than 600 grams [17] and could be used directly in the field for 'point-of-care' monitoring or in decentralised, minimally equipped laboratories.

Several novel diagnostics have been developed for detection of $k d r$ in mosquitoes in attempts to simplify assays and reduce costs, yet TaqMan assays, which use expensive fluorescent probes, remain the most commonly used assay [12]. Melt-curve assays have been previously developed to simultaneously detect both $\mathrm{F}$ and $S$ alleles, which reduces labour and the reagent costs whilst producing easily interpretable results [18]. However, these methods still require substantial upfront costs for equipment and require skilled expertise to perform.

The 1014 assay has been designed so that only a single probe is required to simultaneously discriminate between the L, F and S alleles, whereas the 1575 assay requires a second probe for the detection of the $\mathrm{N}$ and $\mathrm{Y}$ alleles, each using their assay-specific primer pairs. 


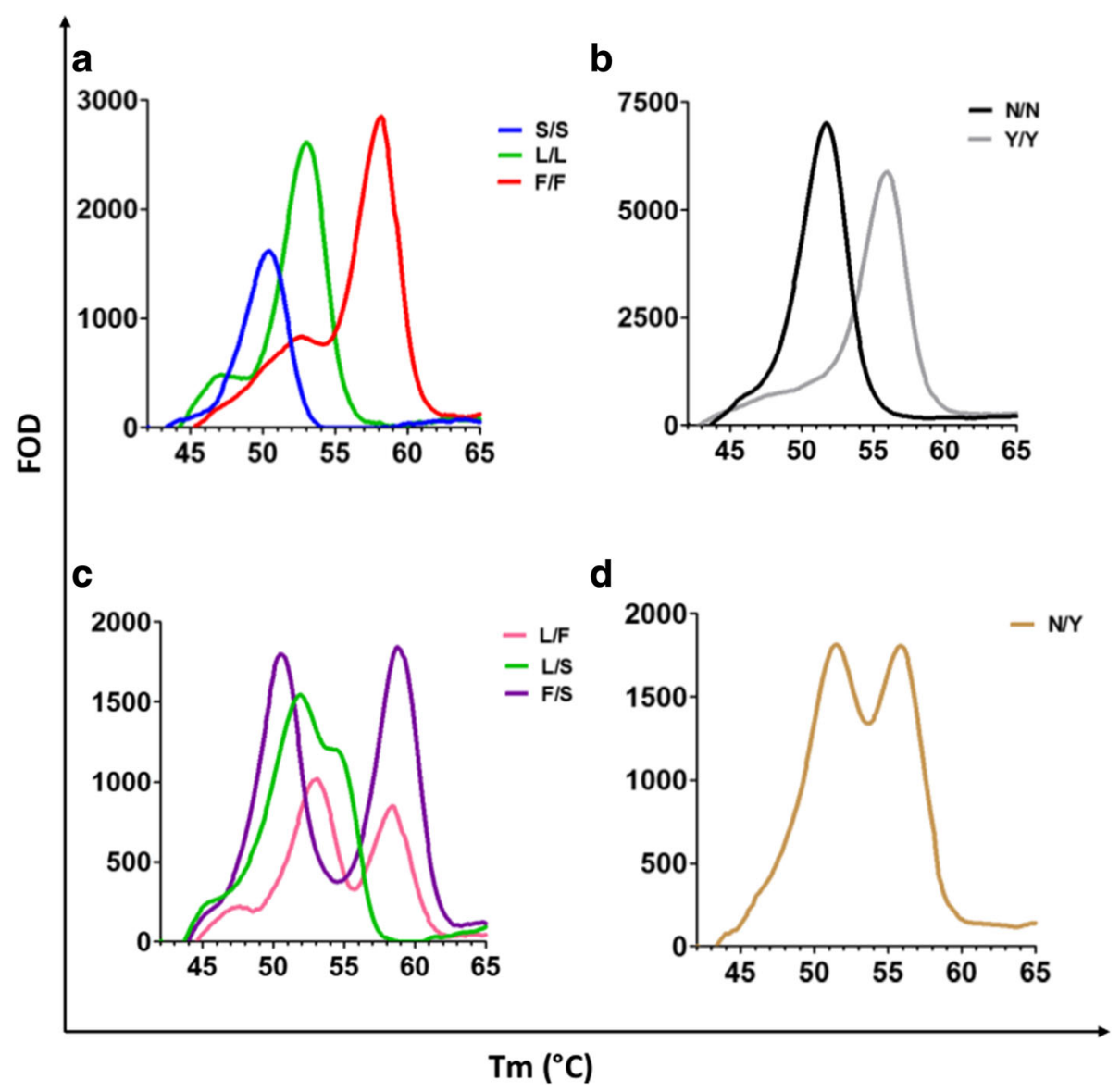

Fig. 2 FOD melt curves of kdr genotypes. FOD melt curves of homozygous genotypes at the 1014 locus (a): S/S (blue), F/F (red) and L/L (WT, green) and at the 1575 locus (b): N/N (WT, black) and N/Y (grey). Heterozygous genotypes are shown in c at the 1014 locus: L/F (pink), L/S (green) and F/S (purple), and $\mathbf{d}$ at the 1575 locus: N/Y (brown)

By comparison, detection of these mutations by conventional TaqMan methods would require 5 different probes: $\mathrm{Y}, \mathrm{F}$ and $\mathrm{S}$ - mutant probes, as well as WT probes for each 1014 and 1575 sequence. The costs of reagents are thus significantly reduced. In addition to the simple workflow and reduced costs on reagents, the cost of the GeneDrive machine itself is estimated to be less than a third of the price of a 48 well rt-PCR machine (US $\$ 19,000-20,000$ [16]).

It should be noted that we observed an increase in peak Tm across 1014F and 1014L genotypes when comparing sample lysates with purified DNA (Table 3). This is most likely due to the differing background of salt

Table 2 Comparison of melting temperature of lysate and extracted DNA template

\begin{tabular}{llllll}
\hline & \multicolumn{2}{l}{ Tm range $\left({ }^{\circ} \mathrm{C}\right)$} & & \multicolumn{2}{l}{ Average Tm \pm SD } \\
\cline { 2 - 3 } \cline { 6 - 6 } Allele & DNA extract $(n)$ & Crude lysate $(n)$ & & DNA extract & Crude lysate \\
\hline $1014 \mathrm{~F}$ & $57.7-58.9(15)$ & $59-59.55(12)$ & & $58.49 \pm 0.39$ & $59.31 \pm 0.14$ \\
$1014 \mathrm{~L}$ & $52.8-53.7(13)$ & $53.9-54.7(11)$ & & $53.4 \pm 0.29$ & $54.29 \pm 0.21$ \\
\hline
\end{tabular}

concentrations in the lysates [19]. For automated Genedrive genotype detection, a defined Tm range would require determination through further testing on different lysates to account for intra-individual variability. Although a larger sample size to determine this would result in a broader Tm range, it is important to note that the Tm shift between each of the alleles remains relatively constant. This could be accounted for in the algorithm for automation of the readout.

The Genedrive cartridge designed here allows for several IR markers to be detected using only two of the three available channels in a cartridge. This allows potential to incorporate a third assay for additional markers or, alternatively the assays could be separated into different cartridges to process multiple samples in one run.

Using pooling strategies, our results show that there is potential to screen DNA from up to 12 mosquitoes for 1014F mutations in a single run (50 $\mathrm{min})$. There is also potential to upscale analysis by increasing numbers of cartridges or machines. Pooling samples for GeneDrive analysis could allow either qualitative detection of the 


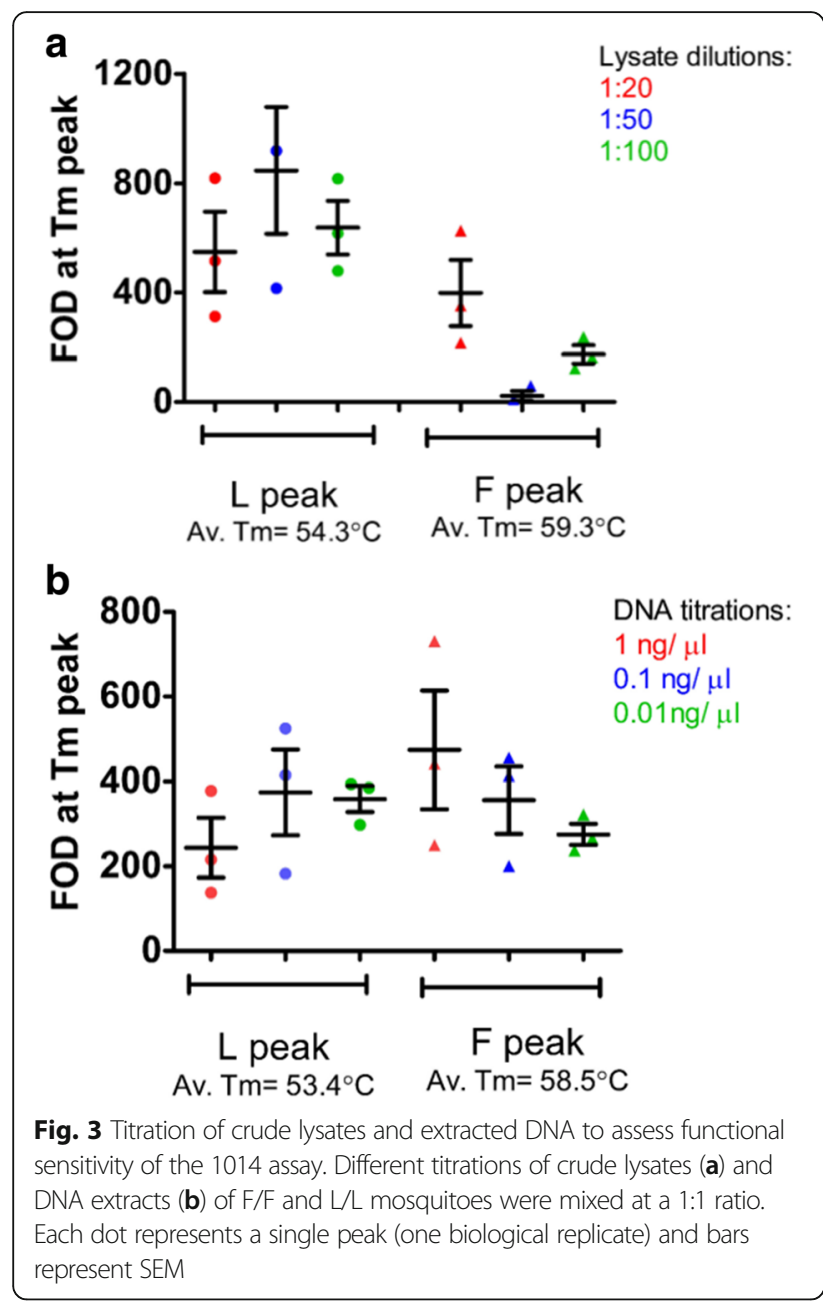

Table 3 Sensitivity and specificity of 1014 and 1575 Genedrive ${ }^{\oplus}$ assays compared to those determined using TaqMan in a test panel of 70 An. gambiae DNA samples

\begin{tabular}{|c|c|c|c|c|c|c|c|c|c|c|c|}
\hline & & & \multicolumn{9}{|c|}{ TaqMan } \\
\hline & & & \multicolumn{6}{|c|}{1014 loci } & \multicolumn{3}{|c|}{1575 loci } \\
\hline & & & $\mathrm{LL}$ & LF & $\mathrm{FF}$ & LS & SS & FS & NN & NY & YY \\
\hline \multirow[t]{9}{*}{ Genedrive $^{\circledast}$} & 1014 loci & $\mathrm{LL}$ & 1 & - & - & - & - & - & - & - & - \\
\hline & & LF & - & 3 & - & - & - & - & - & - & - \\
\hline & & $\mathrm{FF}$ & - & - & 58 & - & - & - & - & - & - \\
\hline & & LS & - & - & - & - & - & - & - & - & - \\
\hline & & SS & - & - & - & - & 1 & - & - & - & - \\
\hline & & FS & - & - & - & - & - & 9 & - & - & - \\
\hline & 1575 loci & NN & - & - & - & - & - & - & 52 & - & - \\
\hline & & NY & - & - & - & - & - & - & - & 14 & - \\
\hline & & $Y Y$ & - & - & - & - & - & - & - & - & 4 \\
\hline
\end{tabular}

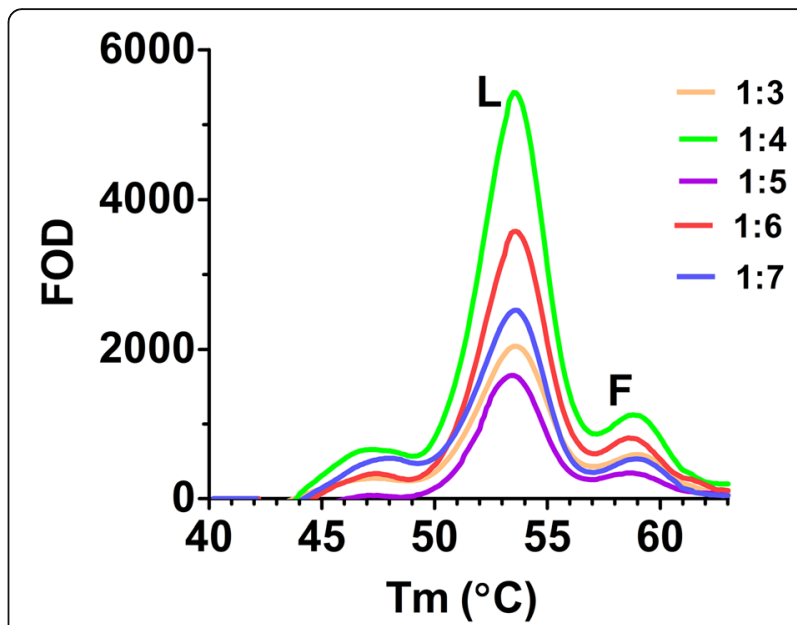

Fig. 4 Pooled DNA from 1014F and WT mosquitoes. DNA was extracted from $F / F$ and $L / L$ mosquitoes and pooled in the following F/F:L/L ratios: 1:3 (orange), 1:4 (green), 1:5 (purple), 1:6 (red) and 1:7 (blue). Each line represents the melt curve of a single replicate

presence of resistance alleles in a population of mosquitoes or quantitative detection of spatial or temporal variation. Pooling samples has the general limitation of making interactions between alleles within loci (dominance effects) and across loci (epistasis) difficult to detect. For the markers considered here, $1014 \mathrm{~F}$ and $1014 \mathrm{~S}$ appear to no more than partially recessive $[20,21]$ and $1575 \mathrm{Y}$ occurs only on a 1014F haplotype [10]. Therefore, useful information can be gained from pooled data, and in terms of vector control management, if a frequency threshold is determined, detection of variation in allele frequencies at resistance phenotype-informative markers from pooled mosquitoes would warrant examination of the insecticide used in a locality. However, for extension to additional markers the importance of dominance level and epistasis must be considered when adopting a pooling strategy.

These assays could be expanded to include different target site mutations and other disease vectors, such as sand flies [22] or triatomine bugs [23], where $k d r$ mutations have also been reported. However, although melt analysisbased assays are useful for the detection of DNA substitutions or indels, it is more difficult to detect multiplication mutations, e.g. duplication of detoxifying enzymes, since melt curve analysis is only semi-quantitative.

\section{Conclusions}

This study describes the development and validation of two simple molecular assays for $k d r$ genotyping in $A n$. gambiae mosquitoes. Our results show accurate detection of the L1014F, L1014S and N1575Y $k d r$-associated SNPs in An. gambiae. Development on the Genedrive ${ }^{\circ}$ platform presents a viable methodology for applying these assays as a field-applicable diagnostic in low-resource settings. 


\section{Abbreviations}

DDT: Dichlorodiphenyltrichloroethane; IRS: Indoor residual spraying; ITN: Insecticide-treated nets; kdr: Knockdown resistance; LLIN: Long-lasting insecticide treated nets; RT-PCR: Real-time polymerase chain reaction; SNP: Single nucleotide polymorphism; Tm: Melting temperature; VGSC: Voltage gated sodium channel

\section{Acknowledgements}

We are grateful to Amy Lynd, Marion Morris and LITE (LSTM) for supply of samples or DNA.

\section{Funding}

This project was funded by contributions from a CiC MRC award (MC PC 14111), an MRC Case studentship award and additional funding from genedrive plc.

\section{Availability of data and materials}

The datasets used and/or analysed during the current study are available from the corresponding author upon reasonable request.

\section{Authors' contributions}

VTU, SA and DW designed the assays in silico. VU optimized and ran the Genedrive $^{\circledast}$ assays and confirmatory TaqMan assays, as well as drafted the manuscript. EHAN collected, extracted and TaqMan-genotyped the mosquitoes from Senegal that were used for control DNA. ER genotyped the blinded panel of samples using the TaqMan assay. SA, DW, MIP and ERA facilitated the collaboration between genedrive plc and the Liverpool School of Tropical Medicine, contributed to writing the manuscript and conceived the overall study design. All authors read and approved the final manuscript.

\section{Ethics approval and consent to participate}

Not applicable.

\section{Competing interests}

This study was performed in collaboration with genedrive plc where SA is currently employed. Genedrive plc were involved in the design and optimisation of the assay but played no role in the analysis of the data, the content of this article or the decision to publish.

\section{Publisher's Note}

Springer Nature remains neutral with regard to jurisdictional claims in published maps and institutional affiliations.

\section{Author details}

${ }^{1}$ Liverpool School of Tropical Medicine, Pembroke Place, L3 5QA, Liverpool, UK. ${ }^{2}$ genedrive plc, Grafton Street, M13 9XX, Manchester, UK. ${ }^{3}$ Université Cheikh Anta Diop, 5005 Dakar, Senegal.

Received: 19 February 2018 Accepted: 9 May 2018

Published online: 18 May 2018

\section{References}

1. World Health Organization. World malaria report. Geneva: WHO; 2013. http://www.who.int/malaria/publications/world_malaria_report_2013/en/

2. Ranson $\mathrm{H}$, Lissenden N. Insecticide resistance in African Anopheles mosquitoes: a worsening situation that needs urgent action to maintain malaria control. Trends Parasitol. 2016:32:187-96.

3. N'Guessan R, Odjo A, Ngufor C, Malone D, Rowland M. A Chlorfenapyr Mixture Net Interceptor ${ }^{\oplus} \mathrm{G} 2$ shows high efficacy and wash durability against resistant mosquitoes in West Africa. PLoS One. 2016;11:e0165925.

4. Dong K, Du Y, Rinkevich F, Nomura Y, Xu P, Wang L, et al. Molecular biology of insect sodium channels and pyrethroid resistance. Insect Biochem $\mathrm{Mol}$ Biol. 2014;50:1-17.

5. Martinez-Torres D, Chandre F, Williamson MS, Darriet F, Berge JB, Devonshire AL, et al. Molecular characterization of pyrethroid knockdown resistance $(k d r)$ in the major malaria vector Anopheles gambiae s.s. Insect Mol Biol. 1998;7:179-84.

6. Davies TG, Field LM, Usherwood PN, Williamson MS. A comparative study of voltage-gated sodium channels in the Insecta: implications for pyrethroid resistance in Anopheline and other Neopteran species. Insect Mol Biol. 2007;16:361-75
7. Gomez SA, Picado A. Systemic insecticides used in dogs: potential candidates for phlebotomine vector control? Trop Med Int Health. 2017;22:755-64.

8. Ranson H, N'Guessan R, Lines J, Moiroux N, Nkuni Z, Corbel V. Pyrethroid resistance in African anopheline mosquitoes: what are the implications for malaria control? Trends Parasitol. 2011;27:91-8.

9. Reimer $\sqcup$, Tripet F, Slotman M, Spielman A, Fondjo E, Lanzaro GC. An unusual distribution of the $k d r$ gene among populations of Anopheles gambiae on the Island of Bioko. Equatorial Guinea. Insect Mol Biol. 2005;14: 683-8.

10. Jones CM, Liyanapathirana M, Agossa FR, Weetman D, Ranson H, Donnelly $\mathrm{MJ}$, et al. Footprints of positive selection associated with a mutation (N1575Y) in the voltage-gated sodium channel of Anopheles gambiae. Proc Natl Acad Sci USA. 2012;109:6614-9.

11. Wang L, Nomura Y, Du Y, Liu N, Zhorov BS, Dong K. A mutation in the intracellular loop III/IV of mosquito sodium channel synergizes the effect of mutations in helix IIS6 on pyrethroid resistance. Mol Pharmacol. 2015;87:421-9.

12. Sarkar M, Baruah I, Srivastava RB, Borkotoki A, Bhattacharyya IK. Highthroughput approach to detection of knockdown resistance $(k d r)$ mutation in mosquitoes, Culex quinquefasciatus, based on real-time PCR using singlelabelled hybridisation probe/melting curve analysis. Pest Manag Sci. 2011;67: 156-61.

13. Genedrive: https://genedrive.com/assays/hcv-assay.php (2016-2017). Accessed 16 Nov 2017

14. Shenai $S$, Armstrong DT, Valli $E$, Dolinger DL, Nakiyingi $L$, Dietze $R$, et al. Analytical and clinical evaluation of the Epistem Genedrive Assay for detection of Mycobacterium tuberculosis. J Clin Microbiol. 2016:54:1051-7.

15. Duffy D, Mottez E, Ainsworth S, Buivan T-P, Baudin A, Vray M, et al. An in vitro diagnostic certified point of care single nucleotide test for IL28B polymorphisms. PLoS One. 2017;12:e0183084.

16. Bass C, Nikou D, Donnelly MJ, Williamson MS, Ranson H, Ball A, et al. Detection of knockdown resistance $(k d r)$ mutations in Anopheles gambiae: a comparison of two new high-throughput assays with existing methods. Malar J. 2007:6:111.

17. Genedrive: https://www.genedrive.com/genedrive-system/documentation php (2016-2017). Accessed 16 Nov 2017.

18. Verhaeghen K, Van Bortel W, Roelants P, Backeljau T, Coosemans M. Detection of the East and West African kdr mutation in Anopheles gambiae and Anopheles arabiensis from Uganda using a new assay based on FRET/ Melt Curve analysis. Malar J. 2006;5:16.

19. Lando DY, Fridman AS, Chang CL, Grigoryan IE, Galyuk EN, Murashko ON, et al. Determination of melting temperature and temperature melting range for DNA with multi-peak differential melting curves. Anal Biochem. 2015; 479:28-36.

20. Lynd A, Weetman D, Barbosa S, Egyir Yawson A, Mitchell S, Pinto J, et al. Field, genetic, and modeling approaches show strong positive selection acting upon an insecticide resistance mutation in Anopheles gambiae s.s. Mol Biol Evol. 2010;27:1117-25.

21. Ramphul U, Boase T, Bass C, Okedi LM, Donnelly MJ, Müller P. Insecticide resistance and its association with target-site mutations in natural populations of Anopheles gambiae from eastern Uganda. TransR Soc Trop Med Hyg. 2018;103:1121-6.

22. Gomes B, Purkait B, Deb RM, Rama A, Singh RP, Foster GM, et al. Knockdown resistance mutations predict DDT resistance and pyrethroid tolerance in the visceral leishmaniasis vector Phlebotomus argentipes. PLoS Negl Trop Dis. 2017;11:e0005504

23. Fabro J, Sterkel M, Capriotti N, Mougabure-Cueto G, Germano M, RiveraPomar $\mathrm{R}$, et al. Identification of a point mutation associated with pyrethroid resistance in the para-type sodium channel of Triatoma infestans, a vector of Chagas disease. Infect Genet Evol. 2012;12:487-91. 